\title{
Mobile Application Integrated with on SAP Business one Software based on Sales and Collection Management System
}

\author{
Udayaprakash. U \\ MCA,UCE(BIT Camps), \\ Anna University, Trichy,Tamilnadu
}

\author{
Dr. S. Sujatha \\ Head of the Department, \\ Department of Computer Application, \\ UCE (BIT Campus),Anna university, \\ Trichy,Tamilnadu
}

\begin{abstract}
The main objective of this project on Mobile Application Integrated with on SAP Business one software based on Sales Management System is to manage the details of Customer, Payment, Sales, Purchasing, Inventory and Supplier. The project is totally built at administrative end and thus only the administrator is guaranteed the access. The purpose of the project is to build an application program to reduce the manual work for managing the Supplier, Payment, Customer, Sales. It tracks all the details about the Sales and inventory.

In today's cooperate world, most of companies work on ERP (Enterprise Resource Planning) systems. These systems are based on local area network. Now if the director of the company wishes to access any data or Reports at remote location, it is not possible. To overcome this problem a new approach called Android based ERP system is presented here, Which would help to maintain the data of ERP at centralized location and can be accessed from anywhere in the world through Android Application. This would help the director to get live updates of company at any location. Moreover this system would also provide a facility of tracking of persona example, sales person using GPS. The location of this person would be sent to the server where we can track them on Google maps. Also the employee of company can be able to insert the details of ERP modules through Android application
\end{abstract}

Keywords - SAP (System Applications and Products), Mobile Application, ERP (Enterprise Resource Planning), GPS (Global Positioning System)

\section{INTRODUCTION}

SAP: SAP Business One is an integrated business management application designed from the ground up for small and midsize businesses like yours. This book provides a complete overview of how you can work with SAP Business One and how it can help your company grow by automating its operations, such as management, business intelligence, sales, purchasing, production, logistics, and financial processes.

\subsection{SAP Business One}

Unlike most other software applications that focus on a specific area of business, such as accounting or customer relationship management (CRM), SAP Business One is an integrated business management application. It offers a complete set of core functions, including financials, customer relationship management, inventory management, sales, purchasing, operations, and logistics, which address the business management needs of your entire company.

SAP Business One is more than the typical financials software that was born out of accounting and bookkeeping, or a standalone customer relationship management solution. It helps businesses like yours manage the entire process of selling and servicing clients, and once orders are taken, they flow directly into automated fulfilment. As a comprehensive and integrated system, SAP Business One gives you full visibility into your business, through on demand analytics in a personalized cockpit, live alerts and approval procedures, and end-to-end business processes.

A process gap exists when manual business tasks are not automated, or automation stops in one application and must be manually transferred to another. Process gaps are resolved by duplicate re-entry of information from one application into another or by constructing brittle, special-purpose software to do the transfer. Process gaps slow a company down, retard change, and reduce the possibilities for automation. Effective and complete integration, in which information flows from one step to the next, bridges the process gap, as shown in

\subsection{The Basics of Financial Accounting}

SAP Business One delivers the tools and reporting capacity you need to manage your company's finances effectively and according to Generally Accepted Accounting Principles (GAAP). SAP Business One can bring your financial accounting to a new level of automation: 
Real-time, really. Every time you add (or post) a transaction, your general ledger is updated. No need to batch journal entries for later posting-unless that's what you want to do.

Automatic journal entries. Perhaps the largest productivity boost in SAP Business One is its ability to create various journal entries automatically — greatly reducing the need to make journal entries or corrections manually.

Drill-down functionality. SAP Business One gives you the ability to drill down using the orange navigation arrows, not only to the general ledger but all the way to the source documents of a transaction - another powerful labour- and time-saving feature.

Task automation. You can automate redundant tasks by using recurring postings and avoid posting mistakes by using posting templates. "webers/m2." Spell units when they appear in text: “...a few henries," not "...a few H."

\section{Existing Work:}

This process already integrated with SAP Business one software in System. This process is more expensive to manage all services for SAP software. Mobile Application for User Registration and Select the Root of the Day and Sales Order and Collection and extra work for Return of the products.

Proposed Work: SAP Business one software process is very complicated to managing all services so to modify for every one easily access to managing of all services. This software is more expensive so every process is very important do not any mistake in Add on Services. So I created on Add on for mobile integration and Database Services and Account Maintained. And Show the customer details of current location on Sales Employee in the Mobile Application. The scope this provide user efficient working environment and more output can be generated through this. This system provides user friendly interface resulting in knowing each and every usability features of the system. This system helps in tracking records so that past records can be verified through them and one can make decisions based on the past records. This system completes the work in a very less time resulting in less time consumption and high level of efficiency. This system is developed in such a way that even a naïve user can also operate the system easily. The calculations are made very quickly and the records directly saved into databases and the databases can be maintained for a longer periods of time. Each record can be retrieved and can be verified for the future transaction. Also this system provides high level of security for data leaking as only admin people can access the databases no changes can be made in it until it verifies the user login id and password.

\section{System Design:}

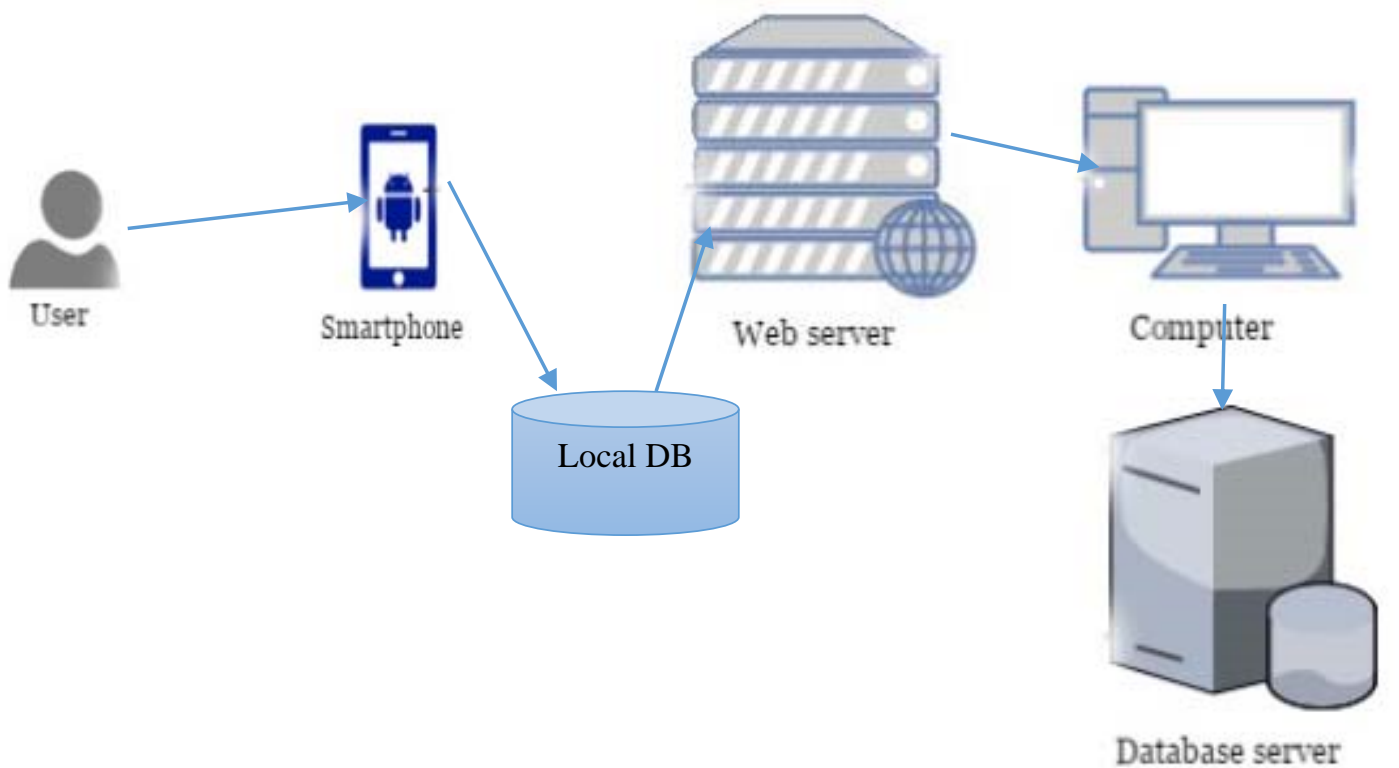

Fig4.1 Work flow of process

The user connects to the Mobile Application for generate sales order stored for Local DB move to web services to communicate computer to store the data into the database. The web browsers communicate with the system through HTTP protocols such as local host. This webserver communicates with the database which can be stored in another server 


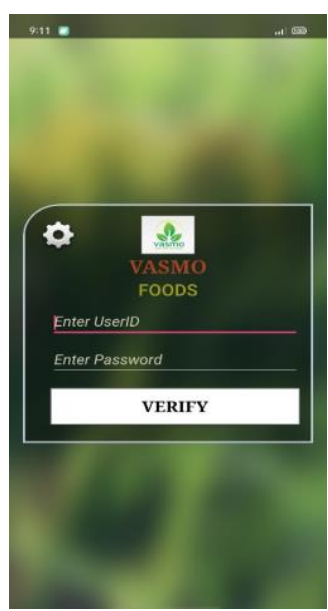

Login page

IMPLEMENTATION SCREENSHOTS.

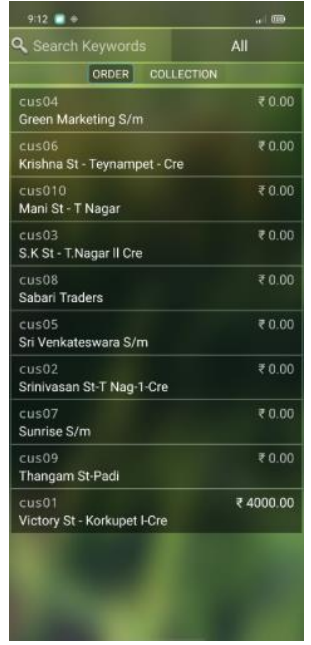

Customer List

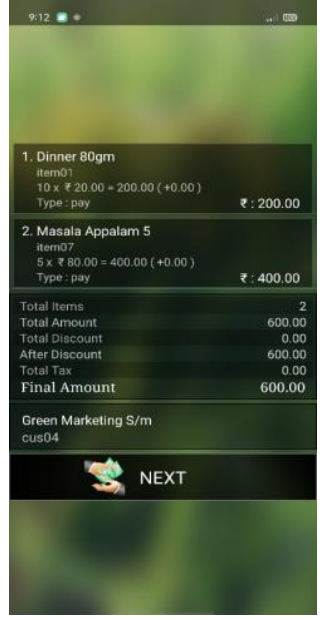

Order process

\section{SAP Process:}

\section{Purchase order in SAP automatically from mobile}

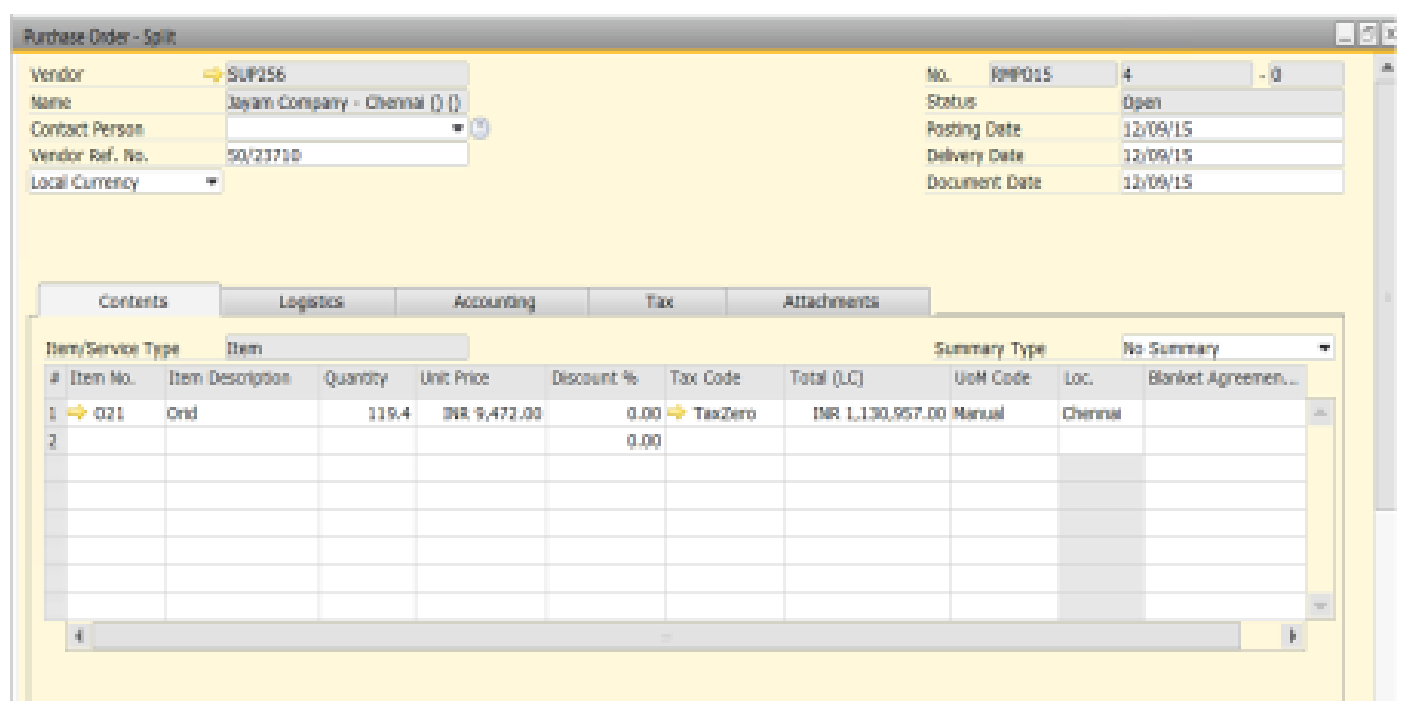

\section{REFERENCE:}

[1] SAP Business One 9.2, version for SAP HANA Document Version: 1.0 - 2017-02-17

[2] J. Wan, S. Tang, Z. Shu, D. Li, S. Wang, M. Imran, and A. Vasilakos, "Software-de ned industrial Internet of Things in the context of industry 4.0," IEEE Sensors J., vol. 16, no. 20, pp. 7373 7380, Oct. 2016.

[3] X. Li, D. Li, J. Wan, A. Vasilakos, C. Lai, and S. Wang, “A review of industrial wireless networks in the context of industry 4.0,"Wireless Netw., pp. 1 19, Nov. 2015, doi: 10.1007/s11276-015-1133-7.2015.

[4] M. Chen, Y. Zhang, L. Hu, T. Taleb, and Z. Sheng, "Cloud-based wireless network: Virtualized, recon gurable, smart wireless network to enable 5G technologies," Mobile Netw. Appl., vol. 20, no. 6, pp. 704 712, 2015.

[5] M. Chen, S. Mao, and Y. Liu, "Big data: A survey," Mobile Netw. Appl., vol. 19, no. 2, pp. 171209 , Apr. 2014. 